\title{
The Use of Magnetic Resonance Angiography in Vascularized Groin Lymph Node Transfer: An Anatomic Study
}

\author{
Joseph H. Dayan, MD ${ }^{1, *}$ Erez Dayan, MD ${ }^{1} \quad$ Alexander Kagen, $\mathrm{MD}^{2} \quad$ Ming-Huei Cheng, MD, MBA ${ }^{3}$ \\ Mark Sultan, MD ${ }^{1}$ William Samson, MD ${ }^{1}$ Mark L. Smith, MD ${ }^{1}$
}

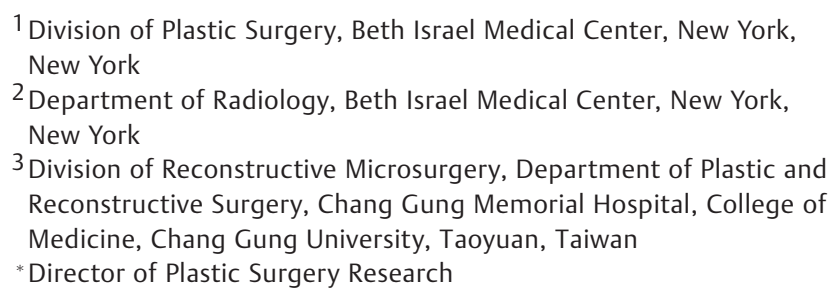

Address for correspondence Joseph H. Dayan, MD, Director of Plastic Surgery Research, Beth Israel Medical Center, 10 Union Square East, Suite 2L, New York, NY 10003 (e-mail: joseph.dayan.md@gmail.com).

J Reconstr Microsurg 2014;30:41-46.

\begin{abstract}
Keywords

- vascularized lymph node transfer

- magnetic resonance angiography

- lymphedema

- lymphatic anatomy

- superficial circumflex iliac artery

- groin flap

Vascularized groin lymph node transfer (VGLNT) has been successfully used to treat lymphedema. However, lack of familiarity with the inguinal node anatomy and concerns regarding donor site morbidity have limited its widespread use. The purpose of this study was to use magnetic resonance angiography (MRA) to clarify the inguinal anatomy and provide a reliable method for identifying the location of the superficial transverse inguinal lymph nodes. In this study MRA was used to evaluate the superficial inguinal lymph nodes in 117 patients. Coordinates of lymph nodes were plotted relative to an axis from the anterior superior iliac spine (ASIS) to the pubic tubercle (PT). The nodes were also plotted relative to the superficial circumflex iliac vein (SCIV) and superficial inferior epigastric vein (SIEV). A total of 1,938 lymph nodes were identified. These lymph nodes were concentrated on one-third the distance from the PT toward the ASIS and $3 \mathrm{~cm}$ perpendicularly below this line. About $67 \%$ of the superficial inguinal nodes were located within the bifurcation of the SIEV and SCIV. The results from this study provide useful guidelines for locating lymph nodes targeted for VGLNT.
\end{abstract}

Lymphedema is one of the most dreaded sequelae of breast cancer treatment. ${ }^{1-4}$ Decongestive physiotherapy and lifelong compression of the upper extremity has been the standard of care. However, compliance is difficult and many patients still have progression of their disease and episodes of cellulitis despite strict adherence to their regimen.

Vascularized groin lymph node transfer (VGLNT) has recently gained increasing popularity among microsurgeons as successful use of this technique has been reported in the treatment of lymphedema. ${ }^{5-9}$ VGLNT involves harvest of lymph nodes and perinodal fat based on the superficial

received

May 27, 2013

accepted after revision

June 30, 2013

published online

September 9, 2013 circumflex iliac artery (SCIA). These lymph nodes typically drain lymph from the lower abdomen. This flap is transferred to the affected extremity with a venous and arterial anastomosis. Induction of lymphangiogenesis into the transplanted nodes with shunting of lymph into the venous circulation is one hypothesis that has been proposed, although a definitive explanation of the underlying mechanism has yet to be established. $5,7,10$

While an increasing number of microsurgeons are performing this technique, there is sparse literature on the superficial groin lymph node anatomy as it relates to lymph
Copyright $\odot 2014$ by Thieme Medical Publishers, Inc., 333 Seventh Avenue, New York, NY 10001, USA. Tel: +1(212) 584-4662.
DOI http://dx.doi.org/ 10.1055/s-0033-1351668. ISSN 0743-684X. 
node transfer. ${ }^{11}$ Detailed knowledge of this anatomy is critical in successfully capturing vascularized lymph nodes as well as avoiding lymph nodes draining the lower extremity which may cause donor site lymphedema. ${ }^{12}$ Magnetic resonance angiography (MRA) is routinely used for perforator flap planning in several centers. ${ }^{13,14}$ In preparation of performing the first lymph node transfer in November 2010, the authors used MRA to study the anatomy of the region. The purpose of this study is to clarify the anatomy of the transverse superficial inguinal lymph nodes using MRA to provide practical guidelines during flap design and harvest.

\section{Methods}

MRA was used on 117 patients to evaluate the location of the transverse superficial inguinal lymph nodes. Deep inguinal lymph nodes medial to the femoral vessels and inferior to the groin crease were excluded because these nodes drain the lower extremity and are not targeted for lymph node transfer. ${ }^{8}$ Using coronal sections on MRA, a line was marked from the anterior superior iliac spine (ASIS) to the pubic tubercle (PT) (-Fig. 1). The coordinates of each lymph node were plotted along this " $\mathrm{x}$ " axis and along a line perpendicular to this " $x$ " axis. These coordinates were then averaged to identify the epicenter around which a $3-\mathrm{cm}$ radius was projected. A percentage of the total number of superficial inguinal nodes located within this radius was calculated.

Attention was then turned to the relationship of the superficial inguinal nodes to the superficial inferior epigastric vein (SIEV) and superficial circumflex iliac vein (SCIV). The percentage of lymph nodes located medial to the SIEV, in between the junction of the SIEV and SCIV, and lateral to the SCIV were calculated (-Fig. 2). These landmarks were selected because the ASIS and PT can be identified preoperatively and the SIEV and SCIV are routinely encountered during lymph node harvest and can be easily visualized on preoperative computed tomography angiography or MRA.

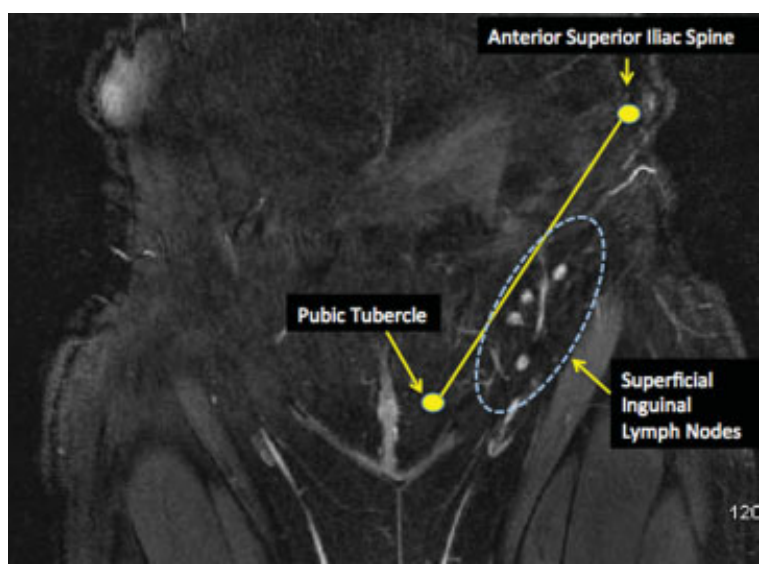

Fig. 1 The magnetic resonance angiography coronal section of left groin. Superficial transverse inguinal lymph nodes are plotted relative to the anterior superior iliac spine-pubic tubercle axis. The vertical chain of lymph nodes located more inferiorly and medially along the femoral vessels was excluded from this study as they drain the lower extremity and should be avoided during lymph node transfer.

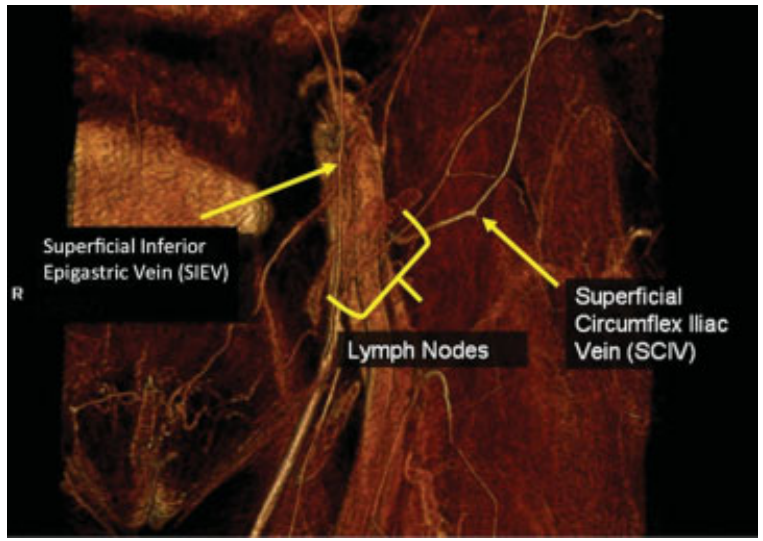

Fig. 2 The magnetic resonance angiography demonstrating the relationship of the superficial inguinal nodes to the superficial circumflex iliac vein and superficial inferior epigastric vein.

\section{Clinical Case and Flap Harvest Technique}

A 53-year-old woman presented with breast cancer-related lymphedema of the left upper extremity. A right groin lymph node flap was designed by palpating the ASIS and PT. A point one-third the distance from the PT toward the ASIS and $3 \mathrm{~cm}$ perpendicularly below this line was marked as the location of the target nodes. This always lies between the inguinal ligament and groin crease. A Doppler was then used to mark the SCIA to confirm the location of the nodes. Last, reverse lymphatic mapping was used as an additional method to confirm the location of the lymph nodes. Technetium was injected preoperatively into the foot and indocyanine green was injected intraoperatively into the lower abdomen. The lymph nodes draining the lower abdomen were visualized and correlated with the SCIAbased nodes. The gamma probe was used to localize the sentinel node draining the lower extremity that was avoided during flap harvest. An incision was made over the SCIA and skin flaps were elevated in the supra-Scarpa plane (-Fig.3). At the superior edge of the flap, the SCIV and SIEV were both identified and divided, with the knowledge that most of the target lymph nodes lay between these

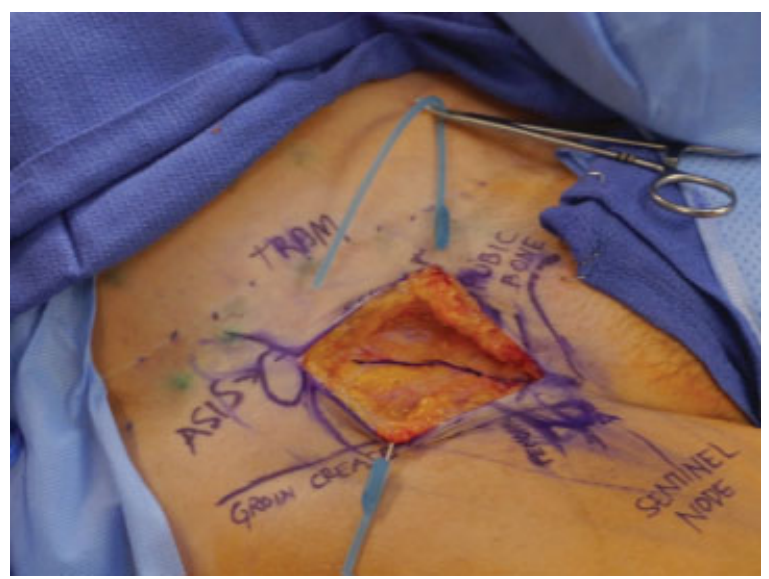

Fig. 3 Vascularized groin lymph node flap harvest. 


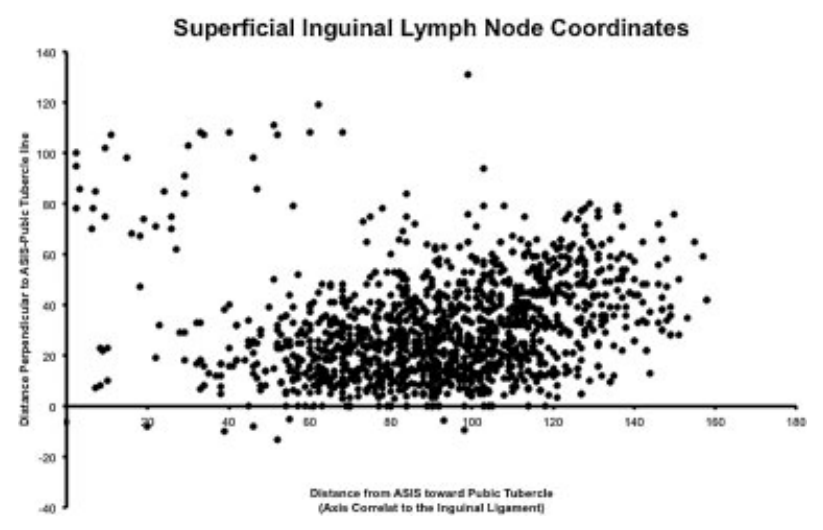

Fig. 4 Location of individual superficial inguinal lymph nodes plotted in relation to the anterior superior iliac spine-pubic tubercle axis.

vessels. The flap was then elevated lateral to medial just above the sartorius fascia. The SCIA branch to the sartorius muscle was divided, and the SCIA and SCIV were mobilized to their origin and harvested for transfer.

\section{Results}

A total of 1,938 distinct superficial inguinal groin lymph nodes were identified. There was an average of 8.2 superficial nodes per groin. All lymph nodes were located deep to Scarpa fascia. The mean distance of the nodes from the ASIS toward the PT was $9.1 \mathrm{~cm}$ (standard deviation [SD] = 2.1) and $3.1 \mathrm{~cm}$ $(\mathrm{SD}=1.9)$ in a perpendicular axis below this line (-Fig. 4 ). This correlates to one-third the distance along the ASIS-PT axis from the PT (-Fig. 5). About $68 \%$ of the superficial inguinal lymph nodes were located within a $3 \mathrm{~cm}$ radius of this epicenter. The superficial nodes tended to cluster at the junction of the SIEV and the SCIV: 67\% within this venous bifurcation, $14 \%$ just inferior to the SCIV, and $19 \%$ medial to the SIEV.

\section{Discussion}

VGLNT is an attractive approach to treating lymphedema in the microsurgical community because it applies techniques familiar to practicing microsurgeons. While VGLNT has been successfully used to treat lymphedema, there are several barriers to its widespread use including lack of familiarity with inguinal node anatomy and concerns regarding donor site morbidity. ${ }^{12}$

While there are several articles that have described the successful use of vascularized lymph node transfer, there is a paucity of literature on the anatomic details of superficial inguinal lymph nodes. ${ }^{5,7,15,16}$ Injection studies on cadavers performed by Assouad et al, confirmed there are superficial inguinal nodes were always present in the angiosome of the SCIA. ${ }^{17}$ Lin et al, describes flap design of the SCIA-based lymph nodes by centering the skin paddle slightly below the inguinal ligament and lateral to the femoral vessels. Cheng has also described a lymph node flap based on medial branch of the common femoral artery. ${ }^{7,17}$ Gharb et al, stressed the

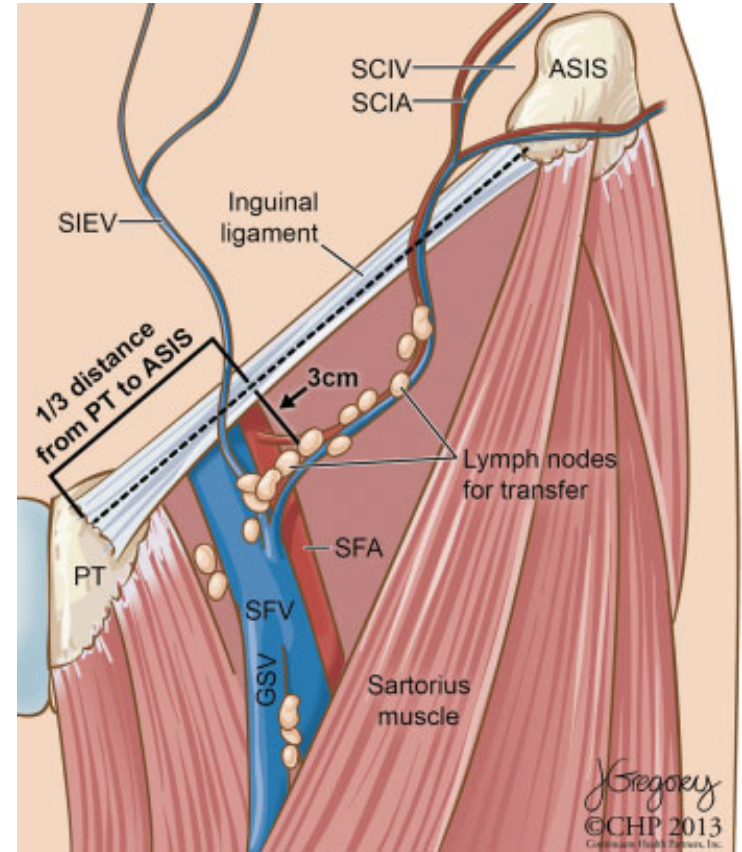

Fig. 5 Illustration clarifying the location of the superficial transverse inguinal lymph nodes in relation to the anterior superior iliac spine (ASIS), pubic tubercle (PT), superficial circumflex iliac vein (SCIV), and superficial inferior epigastric vein (SIEV). The epicenter of these nodes is one-third the distance along the ASIS-PT axis and $3 \mathrm{~cm}$ perpendicularly below this line. These lymph nodes were also concentrated at venous bifurcation of the SCIV and the SIEV.

dominant blood supply to the superficial transverse inguinal nodes is the superficial branch of the SCIA and not its deep branch which dives below the sartorius muscle fascia. ${ }^{18}$ Saaristo and colleagues studied the lymphatic function of the lower extremity following VGLNT using lymphoscintigraphy. ${ }^{19}$ This study concluded there was a mild reduction in lymphatic transport, but no clinical evidence of lymphedema. Based on the location of the sentinel lymph node draining the lower extremity, recommendations were to avoid dissection inferior to the inguinal ligament in the medial femoral region. ${ }^{8}$

One important anatomic detail that may sometimes cause confusion is the location of the SCIA-based lymph nodes relative to the inguinal ligament and the groin crease. In this study, these lymph nodes are consistently located at or just slightly below the inguinal ligament, but above the level of the groin crease. The SCIA itself travels below the inguinal ligament where it originates from the common femoral artery within the femoral triangle. These findings correlate with our observations during lymph node harvest. The authors typically avoid harvesting lymph nodes medial to the SIEV and below the groin crease, consistent with Saaristo recommendations. ${ }^{8}$

Conceptually, VGLNT is a free flap that includes lymph nodes supplied by the SCIA. While this may seem straightforward, the consequences of misguided flap design or lack of detailed understanding of nodal anatomy may result in lower extremity lymphedema. The results in this study provide 


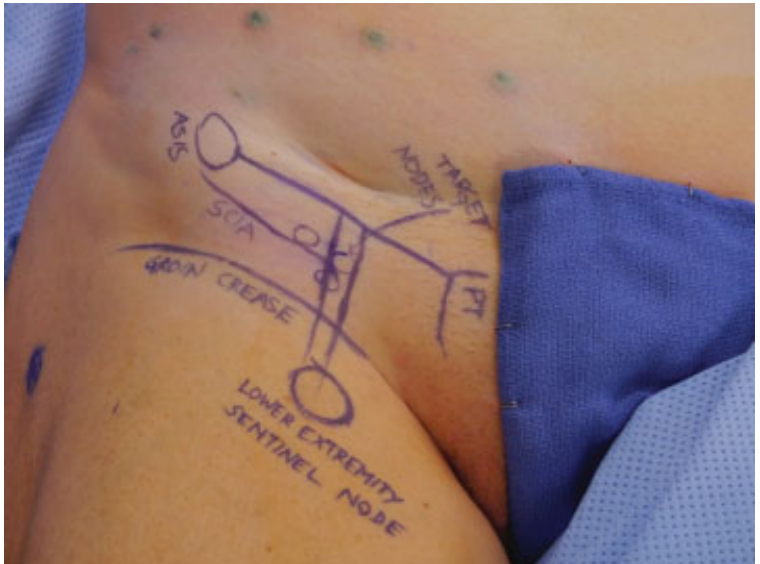

Fig. 6 Preoperative markings using cues from magnetic resonance angiography data, handheld Doppler, indocyanine green injection into the abdomen using the SPY Elite system, and technetium injection into the foot.

practical guidelines for identifying the location of the superficial transverse inguinal lymph nodes both preoperatively and intraoperatively.

The current approach involves the use of four modalities to confirm the anatomic location and physiological drainage patterns of the targeted inguinal lymph nodes. A point onethird the distance from the PT toward the ASIS and $3 \mathrm{~cm}$ below this line along a perpendicular axis is marked as the location of the targeted inguinal nodes, described from the results in this study. A handheld Doppler is then used to localize the superficial circumflex and superficial inferior epigastric vessels. In this practice, both the bony landmarks and vascular landmarks predictably correlate with each other intraoperatively. These methods provide a reliable static anatomic map (-Fig. 6).

The authors have also added two physiological modalities to further validate harvest of the nodes draining the abdomen as well as to avoid lymph nodes draining the lower extremity they have termed reverse lymphatic mapping. This is a modification of Klimberg pioneering method of axillary reverse mapping, but applied to the inguinal region. ${ }^{20-22}$ Indocyanine green is injected intradermally into several areas of the lower abdomen. Using the SPY Elite system (LifeCell Corp., Township of Branchburg, NJ), the lymphatics draining the lower abdomen are visualized and traced to the respective inguinal lymph nodes, confirming these are the nodes of interest. At morning on the day of surgery, the patient also has the ipsilateral foot injected with filtered technetium. Using a gamma probe, the sentinel nodes draining the lower extremity are marked and avoided. All four methodologies consistently correlate with each other in this operative experience. Using a variety of means for identifying the lymph nodes of interest is particularly useful in one's early experience as flap marking and harvest can be executed with greater confidence. Using these modalities, the authors have performed 35 lymph node transfers since November 2010 without any incidence of donor site lymphedema.

\section{Conclusion}

The results of this study provide a practical guide to capturing the superficial inguinal nodes by using identifiable landmarks such as the ASIS, PT, SIEV, and SCIV. MRA is a useful tool in guiding flap design for VGLNT.

\section{Disclosure \\ No Disclosures.}

\section{References}

1 Herd-Smith A, Russo A, Muraca MG, Del Turco MR, Cardona G. Prognostic factors for lymphedema after primary treatment of breast carcinoma. Cancer 2001;92(7):1783-1787

2 Kornblith AB, Herndon JE II, Weiss RB, et al. Long-term adjustment of survivors of early-stage breast carcinoma, 20 years after adjuvant chemotherapy. Cancer 2003;98(4):679-689

3 Ozaslan C, Kuru B. Lymphedema after treatment of breast cancer. Am J Surg 2004;187(1):69-72

4 Schünemann $\mathrm{H}$, Willich $\mathrm{N}$. Lymphoedema of the arm after primary treatment of breast cancer. Anticancer Res 1998;18(3C): 2235-2236

5 Becker C, Assouad J, Riquet M, Hidden G. Postmastectomy lymphedema: long-term results following microsurgical lymph node transplantation. Ann Surg 2006;243(3):313-315

6 Becker C, Hidden G. Transfert de lambeaux lymphatiques libres. Microchirurgie et étude anatomique [Article in French]. J Mal Vasc 1988;13(2):119-122

7 Lin CH, Ali R, Chen SC, et al. Vascularized groin lymph node transfer using the wrist as a recipient site for management of postmastectomy upper extremity lymphedema. Plast Reconstr Surg 2009;123 (4):1265-1275

8 Viitanen TP, Mäki MT, Seppänen MP, Suominen EA, Saaristo AM. Donor-site lymphatic function after microvascular lymph node transfer. Plast Reconstr Surg 2012;130(6):1246-1253

9 Penha TR, Ijsbrandy C, Hendrix NA, et al. Microsurgical techniques for the treatment of breast cancer-related lymphedema: a systematic review. J Reconstr Microsurg 2013;29(2):99-106

10 Tobbia D, Semple J, Baker A, Dumont D, Johnston M. Experimental assessment of autologous lymph node transplantation as treatment of postsurgical lymphedema. Plast Reconstr Surg 2009;124 (3):777-786

11 Vinnicombe SJ, Norman AR, Nicolson V, Husband JE. Normal pelvic lymph nodes: evaluation with CT after bipedal lymphangiography. Radiology 1995;194(2):349-355

12 Vignes S, Blanchard M, Yannoutsos A, Arrault M. Complications of autologous lymph-node transplantation for limb lymphoedema. Eur J Vasc Endovasc Surg 2013;45(5):516-520

13 Mathes DW, Neligan PC. Preoperative imaging techniques for perforator selection in abdomen-based microsurgical breast reconstruction. Clin Plast Surg 2010;37(4):581591, xi

14 Mathes DW, Neligan PC. Current techniques in preoperative imaging for abdomen-based perforator flap microsurgical breast reconstruction. J Reconstr Microsurg 2010;26(1):3-10

15 Chen HC, O’Brien BM, Rogers IW, Pribaz JJ, Eaton CJ. Lymph node transfer for the treatment of obstructive lymphoedema in the canine model. Br J Plast Surg 1990;43(5):578-586

16 Clodius L, Smith PJ, Bruna J, Serafin D. The lymphatics of the groin flap. Ann Plast Surg 1982;9(6):447-458

17 Assouad J, Becker C, Hidden G, Riquet M. The cutaneo-lymph node flap of the superficial circumflex artery. Surg Radiol Anat 2002;24 (2):87-90 
18 Gharb BB, Rampazzo A, Spanio di Spilimbergo S, Xu ES, Chung KP, Chen HC. Vascularized lymph node transfer based on the hilar perforators improves the outcome in upper limb lymphedema. Ann Plast Surg 2011;67(6):589-593

19 Tammela T, Saaristo A, Holopainen T, et al. Therapeutic differentiation and maturation of lymphatic vessels after lymph node dissection and transplantation. Nat Med 2007;13(12):1458-1466

20 Boneti C, Korourian S, Bland K, et al. Axillary reverse mapping: mapping and preserving arm lymphatics may be important in preventing lymphedema during sentinel lymph node biopsy. J Am Coll Surg 2008;206(5):1038-1042, discussion 1042-1044

21 Klimberg VS. A new concept toward the prevention of lymphedema: axillary reverse mapping. J Surg Oncol 2008;97(7): 563-564

22 Thompson M, Korourian S, Henry-Tillman R, et al. Axillary reverse mapping (ARM): a new concept to identify and enhance lymphatic preservation. Ann Surg Oncol 2007;14(6): 1890-1895 